\title{
Self-assembled collagen-like-peptide implants as alternatives to human donor corneal transplantation
}

\author{
Mohammad Mirazul Islam, Ranjithkumar Ravichandran, D. Olsen, Monika Kozak
}

Ljunggren, Per Fagerholm, Chyan-Jang Lee, May Griffith and Jaywant Phopase

\section{Linköping University Post Print}

\section{Tweet}

N.B.: When citing this work, cite the original article.

Original Publication:

Mohammad Mirazul Islam, Ranjithkumar Ravichandran, D. Olsen, Monika Kozak Ljunggren, Per Fagerholm, Chyan-Jang Lee, May Griffith and Jaywant Phopase, Self-assembled collagenlike-peptide implants as alternatives to human donor corneal transplantation, 2016, RSC Advances, (6), 61, 55745-55749.

http://dx.doi.org/10.1039/c6ra08895c

Copyright: Royal Society of Chemistry

http://www.rsc.org/

Postprint available at: Linköping University Electronic Press

http://urn.kb.se/resolve?urn=urn:nbn:se:liu:diva-130324 


\title{
Self-assembled collagen-like-peptide implants as alternatives to human donor corneal transplantation
}

\author{
M. Mirazul Islam, ${ }^{\mathrm{a}, \mathrm{b}, \S}$ R. Ravichandran, ${ }^{\mathrm{c},}$ D. Olsen, ${ }^{\mathrm{d}}$ M. K. Ljunggren, ${ }^{\mathrm{b}}$ Per Fagerholm, ${ }^{\mathrm{b}}$ C. J. Lee, ${ }^{\mathrm{b}, \mathrm{c}}$ \\ M. Griffith ${ }^{\mathrm{a}, \mathrm{b}, \S, *}$ and J. Phopase ${ }^{\mathrm{c}, \S}$
}

Extracellular matrix proteins like collagen promote regeneration as implants in clinical studies. However, collagens are large and unwieldy proteins, making small functional peptide analogs potentially ideal substitutes. Self-assembling collagen-like-peptides conjugated with PEG-maleimide were assembled into hydrogels. When tested pre-clinically as corneal implants in mini-pigs, they promoted cell and nerve regeneration, forming neo-corneas structurally and functionally similar to natural corneas.

\section{Introduction}

Resident stem cells capable of regeneration are present in almost every organ in the body but frequently cannot achieve the repair needed after damage by injury or disease. The limiting factor is often the extracellular matrix (ECM) surrounding the cells. During damage, the ECM is frequently replaced by scar tissue, which does not provide the required structural integrity and inhibits regeneration of functional tissue. ${ }^{1}$ The replacement of scar tissue with ECM or biomaterials that mimic its structure and function could therefore promote regeneration.1,2 Regenerating damaged tissues and organs, including corneas, could potentially mitigate the need for organ transplantation, which currently faces acute worldwide shortage and immune rejection issues.

We have previously shown in human corneal transplantation clinical studies that cell-free corneal ECM mimics made from recombinant human collagen (RHC) stimulated regeneration of the human cornea, an organ that normally does not regenerate on its own. ${ }^{3,4}$ However, RHC replicates human collagen, which, like many other ECM biopolymers, is comprised of large proteins and is difficult to manipulate. Smaller units of complex proteins, particularly those capable of self-assembly have been examined as controllable ECM mimics, as they can form a wide range of structures including nanofibres. ${ }^{5}$ Collagen-like peptides (CLPs), also known as collagen-mimetic peptides (CMPs), have recently been investigated as potential alternatives to collagen, as they can self-assemble and form triple helical nanofibers like collagen. ${ }^{6-14}$ In order to stabilize the triple helices of CLPs, polymer templates that can link the three peptide chains together with sufficient flexibility to allow for proper packing of the chains with correct amino acid register have been tested. ${ }^{7}$ More recent designs have used collagen peptides as the physical crosslinks for the polymer system through triple helix formation..$^{6,7}$ CLPs and CLP-polymer systems have now been tested in vitro as engineered 3D scaffolds on their own, ${ }^{8}$ conjugated to polyethylene glycol (PEG) backbones, ${ }^{9-11}$ and complexed with bioactive factors ${ }^{12,13}$ or localization agents such as gold nanoparticles. ${ }^{14}$ However, the majority of studies have been confined to in vitro testing with cells.

The trend in regenerative medicine is now towards the use of complex, naturally derived ECM macromolecules, as intact decellularised scaffolds or as processed and purified concentrated liquids or finely ground powder that are then reconstituted into hydrogels. ${ }^{2}$ Here, we report on the capacity of a CLP-PEG based hydrogel to serve as a functional analog to full-length collagen for promoting regeneration, using the corneal transplantation as a test bed.

\section{Results and discussion}

In 2011, O'Leary et al. described a 36 amino acid peptide, (Pro-Lys-Gly)4(Pro-Hyp-Gly)4(Asp-Hyp-Gly)4 that mimicked collagen fibrils. ${ }^{8}$ As described, this CLP was only able to self-assemble into a soft hydrogel, while clinical use in corneal transplantation requires implants to be sufficiently robust for surgical handling. Collagen is a large trimeric protein (approx. $300 \mathrm{kDa}$ ) comprising a long backbone chain (approx. 1000 amino acids per strand) that imparts high mechanical strength. Here, we modified the O'Leary CLP by the addition of two extra amino acids - a glycine spacer and a cysteine to allow covalent attachment of an 8-armed 
polyethylene glycol maleimide as described by Perez et al. ${ }^{10}$ The presence of a multi-arm template provided an adequate balance between rigidity and flexibility. As CLP self-assembled into triple helices, conjugation with PEG resulted in a higher order supramolecular self-assembly that was then crosslinked with $\mathrm{N}$-(3dimethylaminopropyl)-N'-ethylcarbodiimide (EDC) to form a stabilized cornea shaped and sized implant $(12 \mathrm{~mm}$ in

a
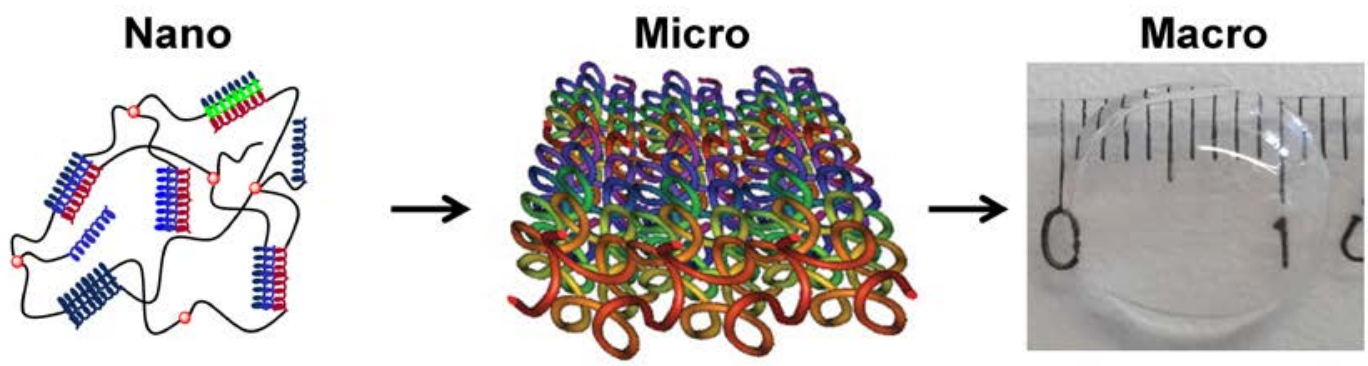

b

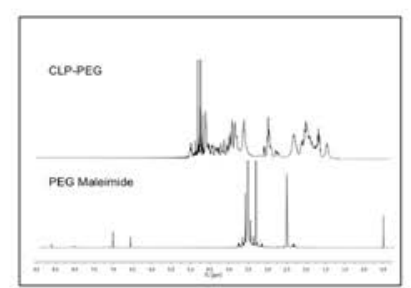

C

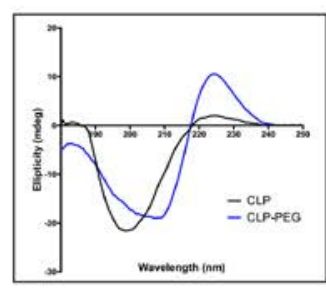

d

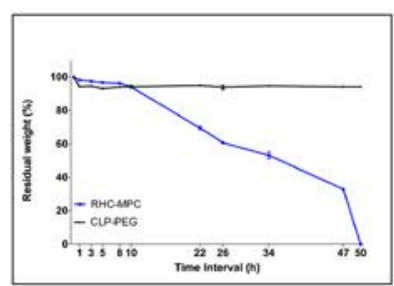

e

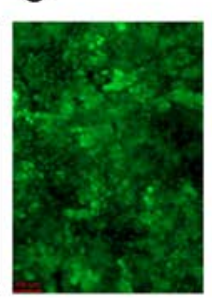

f

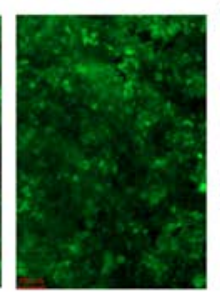

g

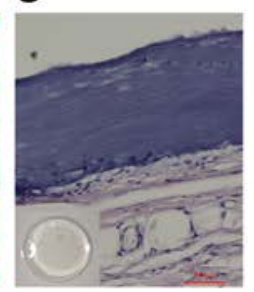

Fig. 1 Fabrication and characterization of CLP-PEG implants. (a) Schematic showing the assembly of CLP-PEG from the nanoscale into macroscale corneal shaped hydrogels. Eight CLP chains are attached to 8-arms of PEG-maleimide. When CLP self-assembles into triple helices at nano level, PEG-maleimide is drawn together, triggering further self-association into nanofibrils. Subsequently higher order supramolecular assembly within a mold and EDC crosslinking results in the formation of a cornea-shaped implant. (b) NMR spectra of CLP-PEG and PEG maleimide, showing maleimide peaks at $\delta 6.5-7.0 p p m$ that disappeared after conjugation of CLP. (C) CD spectra of CLP and CLP-PEG confirming their collagen-like triple helical secondary structure. The larger positive peak at $221 \mathrm{~nm}$ for CLP-PEG compared to CLP only shows a higher order triple helical propensity facilitated by the presence of the PEG backbone. (d) CLP-PEG is resistant to in vitro biodegradation in high concentrations of collagenase, compared to RHC-MPC, which degraded completely over 50 hours. (e) Human corneal epithelial cells after 5 days in culture on CLPPEG hydrogels, stained with Live/Dead stain. Green, live cells; red, dead cells. Cells are proliferating and healthy and starting to stratify, as with cells growing on RHC-MPC control hydrogels (f). (g) Representative H\&E stained section of a CLP-PEG hydrogel implanted subcutaneously into a rat for 90 days, showing a smooth, intact material edge and absence of heavy fibrotic tissue or inflammatory cells. Inset: Intact 10 mm diameter,

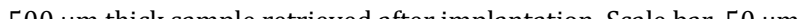

diameter, $500 \mu \mathrm{m}$ thick) comprising CLP and PEG-maleimide (CLP-PEG hydrogels) (Fig. 1a). See Supplementary Methods online.

CLP conjugation to PEG-maleimide was confirmed by 1H-NMR (Fig. 1b). Circular Dichroism (CD) spectroscopy analysis of CLP-PEG showed increased triple helical propensity at $221 \mathrm{~nm}(\mathrm{Rpn}=0.55)$ compared to CLP alone (Rpn=0.093) (Fig. 1c). The PEG template had likely enforced proximity of peptide strands for intramolecular assembly, facilitating the intermolecular, collagen-like higher order triple helical association at room temperature.

EDC-crosslinked CLP-PEG hydrogels were optically transparent, but significantly mechanically weaker than human corneas since they comprise over $90 \%$ water (human corneas only have $78 \%$ water (Table 1)). However, they were sufficiently robust to retain their shape (Fig. 1a). Furthermore, the hydrogels were stable and did not biodegrade rapidly when exposed to the artificially high concentrations of collagenase $(5 \mathrm{U} / \mathrm{ml})$ used to simulate an accelerated biodegradation process. The CLP-PEG hydrogels showed minimal degradation in comparison to hydrogels comprising interpenetrating networks of RHC-2-methacryloyloxyethyl phosphorylcholine (RHC-MPC) implants that remained stable in the severely pathologic eyes (Fig. 1d). Human corneal epithelial cells, when seeded onto CLP-PEG implants, showed good proliferation without any cytotoxic effects (Fig. 1e) comparable to that on RHC-MPC hydrogels (Fig. 1f). This indicated good in vitro biocompatibility. Subsequent subcutaneous implantation of CLP-PEG hydrogels into the dorsum of rats for 90

Table 1. Properties of CLP-PEG corneal implants in comparison to human corneas.

\begin{tabular}{|c|c|c|c|c|c|c|}
\hline Properties & $\begin{array}{l}\text { Transmissio } \\
\mathrm{n} \\
(\%)\end{array}$ & $\begin{array}{l}\text { Backscatte } \\
\text { r } \\
(\%)\end{array}$ & $\begin{array}{l}\text { Tensile } \\
\text { Strength } \\
\text { (MPa) }\end{array}$ & $\begin{array}{l}\text { Elongation } \\
\text { (\%) }\end{array}$ & $\begin{array}{l}\text { Modulus } \\
\text { (MPa) }\end{array}$ & $\begin{array}{l}\text { Water content } \\
(\%)\end{array}$ \\
\hline CLP-PEG & $92.4 \pm 0.95$ & $\begin{array}{ll}0.90 & \pm \\
0.17 & \\
\end{array}$ & $0.07 \pm 0.02$ & $58.30 \pm 4.49$ & $0.18 \pm 0.06$ & $91.65 \pm 1.10$ \\
\hline $\begin{array}{l}\text { Human } \\
\text { Cornea } 3\end{array}$ & $87.1 \pm 2.0$ & $<3$ & $3.81 \pm 0.40$ & - & $3-13$ & 78 \\
\hline
\end{tabular}


days (after ethical approval from the local ethical committee, Linköpings Djurförsöksetiska Nämnd, and in compliance with the Swedish Animal Welfare Ordinance and the Animal Welfare Act) confirmed their biocompatibility in vivo (Fig. 1g) as the implants were relatively intact, and remained free of immune cells or thick fibrotic encapsulation after 90 days in vivo.

In preparation for clinical translation, CLP-PEG hydrogels were tested for potential use in transplantation as an alternative to donated human corneas, in cases where the endothelium is healthy. The cornea is the transparent front part of the eye, which acts as a lens to direct light to the back for vision. Permanent transparency loss results in blindness, which can be treated by transplantation of donor tissue. However, there is a severe worldwide donor shortage, and not all conditions are amenable to donor grafting. ${ }^{15}$

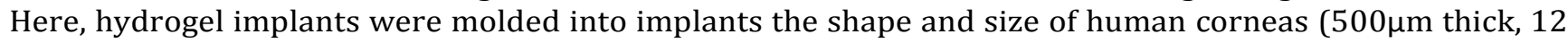
mm diam). In compliance with the OECD Principle of Good Laboratory Practice (GLP), ENV/MC/CHEM (98) 17, 1997, and with local ethical permission from Stockholms Norra Djurförsöksetiska Nämnd, implants was grafted into the corneas of four Gottingen mini-pigs by anterior lamellar keratoplasty, after excision of the pigs own corneal tissues. At 12 months post-implantation, CLP-PEG implants remained stably incorporated and optically clear, as judged by slit lamp biomicroscopy, within the pigs' eyes (Fig. 2a), similar to the contralateral unoperated control corneas (Fig. 2b). However, mild haze was observed at the material-host interface. The optical transparency allowed for real-time imaging by in vivo confocal microscopy of corneal epithelial cells growing over the implants and in-growth of corneal stromal cells into the

implants (Supplementary Fig. 1). Histologically, the regenerated neo-corneas (Fig. 2c) resembled their contralateral unoperated controls (Fig. 2d). Each cell-free CLP-PEG implant had been replaced by a tissue comprising an epithelium and underlying stroma with similar morphology to the healthy control corneas. In vivo confocal microscopy also showed that corneal nerves had grown into the implants, forming a subepithelial nerve plexus comprising parallel nerves (Fig. 2e), resembling the pattern seen in their healthy, unoperated, contralateral counterparts (Fig. 2f). Biochemical analyses of grafted CLP-PEG implants samples showed the presence of corneal collagens type I and V, which were not present in the initial implants (Fig. 2g,

a
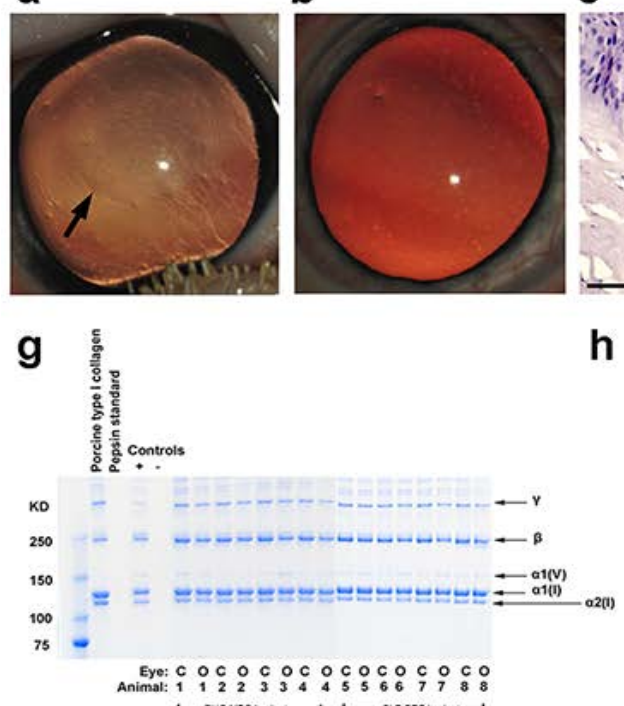

-PACUPCing

b

h
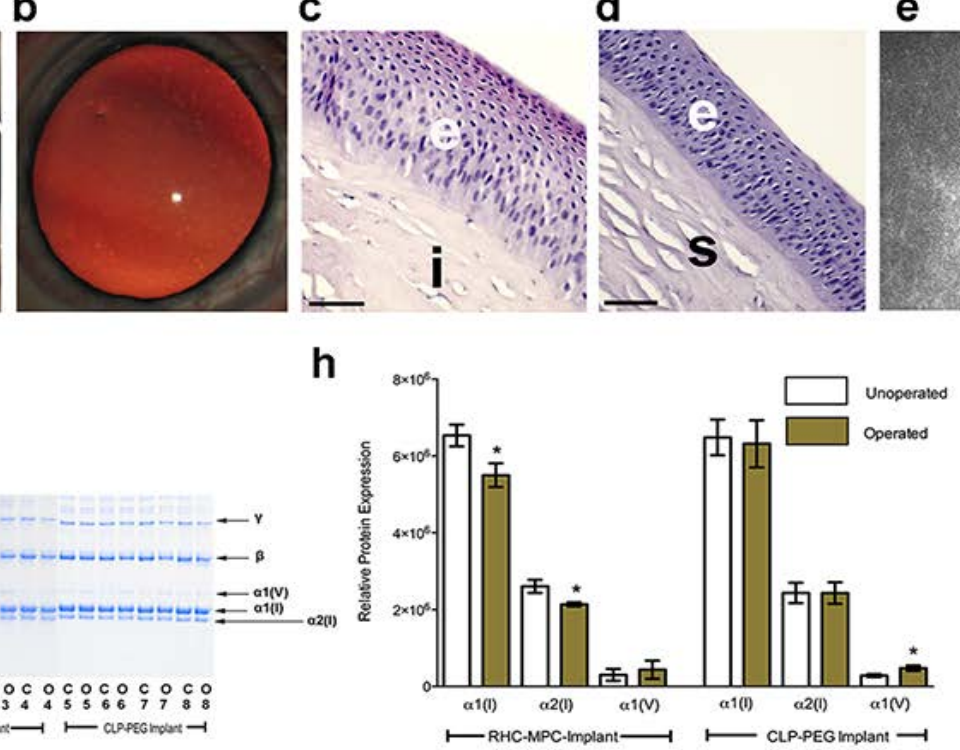

d
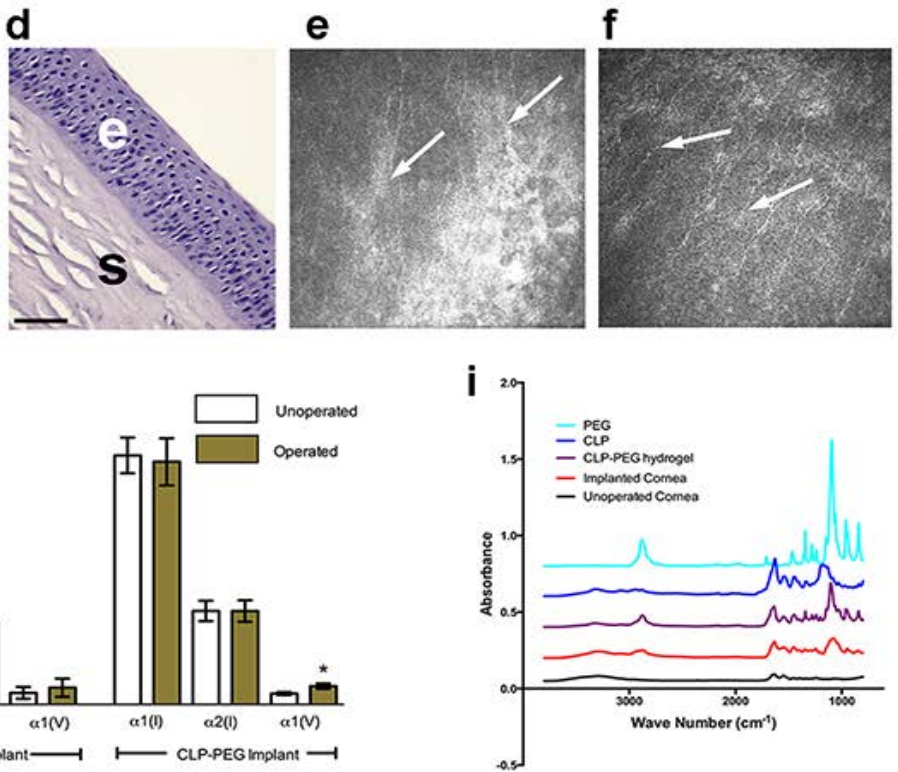

Figure 2. Post-surgical corneal regeneration at 12 months after CLP-PEG implantation. (a) Optically clear CLP-PEG implant (arrowed) stably integrated within the pig cornea, compared to its healthy, unoperated contralateral cornea (b). Blood vessels are seen in the implanted cornea but stop at the margin of the implant. (c) H\&E staining of a representative regenerated CLP-PEG neo-cornea compared to a healthy control cornea (d), showing similar morphology. e, epithelium; s, stroma; i, implant. Scale bars, $50 \mu \mathrm{m}$ (e) In vivo confocal microscopy shows the regenerated nerve (arrows) in CLP-PEG cornea, that follow a parallel pattern similar to that of the unoperated cornea (f). (g) SDS-PAGE separated proteins from the central cornea area of both implanted and control corneas shows the presence of $\alpha 1$ and $\alpha 2$ chains for type I collagen ( $\alpha 1$ (I) and $\alpha 2$ (I), respectively) and $\alpha 1$ chain for type $V(\alpha 1(\mathrm{~V}))$, in the regenerated CLP-PEG implanted cornea, similar to RHC-MPC and the unoperated healthy control corneas. (h) Analysis of normalized, relative protein content shows that the levels of $\alpha 1(\mathrm{I})$ and $\alpha 2(\mathrm{I})$ in CLP-PEG implanted corneas were similar to that of the control healthy corneas (no statistical significance) while the level of $\alpha 1(\mathrm{~V})$ however was higher than that of the control (P $\leq 0.05$, indicated by $*$ ). In RHC-MPC implanted corneas, levels of both $\alpha 1(\mathrm{I})$ and $\alpha 2(\mathrm{I})$ were significantly lower than in the unoperated controls $\left({ }^{*}\right)$, while $\alpha 1(\mathrm{~V})$ were similar. No differences were observed between RHCIII-MPC and CLP-PEG implants in general. (i) FTIR analysis of regenerated pig neo-corneas 12 months after grafting with CLP-PEG implants shows the presence of both CLP and PEG. 
h). This profile was similar to that of RHC-MPC implants that were followed longitudinally as benchmarks. More importantly, the collagen profiles were similar to those of healthy, control corneas albeit differing in the quantities of collagen. This showed that remodeling of the initial fully synthetic implant had occurred. However, FTIR spectroscopy revealed CLP and PEG profiles in the implanted area (Fig. 2i), indicating remodeling was incomplete and likely still on-going at 12 months post-operation. Overall, the implanted corneas did not differ significantly in thickness from unoperated controls. This is most likely due to the formation of neo-corneal tissue that allows for modulation of proper corneal hydration.

A range of self-assembling amphiphilic peptides, including

CLPs has now been examined as scaffolds for promoting corneal regeneration since they can form a wide range of structures including nanofibers like ECM and they are easily tunable to support adhesion and proliferation of corneal cells. ${ }^{16,17}$ ECM-derived cell adhesion peptides containing the laminin motif, Tyr-Ile-Gly-Ser-Arg, or the fibronectin motif, Arg-Gly-Asp, have been tested as injectable scaffolds and shown to promote regeneration of the corneal stroma in rabbits. ${ }^{18}$ However, in many corneal pathologies, the tissue is scarred leading to loss of transparency and blindness. Excision of the scarred region and replacement with a robust hydrogel that could act as a corneal substitute is therefore necessary. Hence, conjugation to a polymer backbone is needed.

The most convenient method for fabrication of peptide-synthetic polymer conjugates is to couple a peptide that is functionalized (either at the termini or side chain) with a complementary functionalized synthetic polymer. ${ }^{6}$ While a number of synthetic polymers have been explored as backbones for peptides, many of the successful reported hybrids have incorporated PEG and its derivatives. ${ }^{6,7}$ CLP-PEG-maleimide hydrogels that were similar to ours, reported by Perez et al., were thermoresponsive and formed a liquid-like state close to the melting temperature of the CLPs. ${ }^{11}$ Here, a further crosslinking step using EDC and its co-reactant, $\mathrm{N}$ Hydroxysuccinimide (NHS) allowed us to fabricate CLP-PEG hydrogels that remained in an irreversible colloidal state. This is important as implants meant to replace pathologic tissue need to remain in place as scaffolds to permit regeneration and remodeling by incoming cells until complete formation of new tissues has occurred. The use of PEG may also serve a second function promoting immune tolerance of the implants, as conjugation of protein-based drugs has been shown to reduce immunogenicity. ${ }^{19}$

In the present study, CLP peptide conjugated with PEG-maleimide resulted in a transparent corneal implant that was sufficiently robust for transplantation. The implant showed stable and functional integration, promoting regeneration of corneal epithelial and stromal tissue and ECM components, as well as nerves in a similar manner to RHC-MPC implants that have now been clinically tested. ${ }^{3}$ Like RHC-MPC, the peptide-PEG hydrogel most likely served as a physical scaffold that provided a microenvironment that was conducive to ingrowing cells to proliferate, differentiate and to synthesize ECM components, resulting in a neo-cornea. For translation into clinical application, the use of synthetic peptide analogs in place of animal extracted collagen allows for lot-to-lot homogeneity in raw materials as well as circumventing potential transmission of viruses and other pathogens. As a replacement of RHC, CLP-PEG is potentially easier to manipulate and would allow for future functionalization.

\section{Conclusions}

We have shown that self-assembling CLP-PEG successfully mimicked the function of full-length recombinant human collagen as corneal implants, promoting stable regeneration of several cell types in appropriate configurations. All regenerated neo-corneas were optically transparent, and based on early results, allowed the restoration of morphological features approximating those of natural corneas, suggesting their future applicability for clinical grafting. More extensive analyses of the implants are required to determine the extent to which these CLP-PEG implants were able to mimic hydrogels made from full-length collagen, and to elucidate possible mechanisms of collagen-induced regeneration. Furthermore, the performance of these implants in human patients remains to be determined. Nevertheless, we have demonstrated the potential utility of CLP-based hydrogels for future clinical use.

\section{Acknowledgements}

This work was funded by a Vinnova Indo-Sweden grant (dnr 2013-04645), the Integrative Regenerative Medicine Centre, Linköping University (LiU) and Region Östergötland. RR was supported by a Swedish Research Council grant (dnr 621-2012-4286). The content of the manuscript is disclosed in unpublished patent applications PCT/EP2015/073010, GB1506316.7 (J Phopase, inventor; Ferentis, assignee). We thank Dr. Philip Lewis, Cardiff University, UK, for his help in characterising the CLP-PEG hydrogels and Adlego AB, Sweden, for conducting the GLP mini-pig study. MG acknowledges her appointment as visiting professor at the 
Tokyo Dental College Ichikawa General Hospital, Japan, and affiliated researcher at Maisonneuve-Rosemont Hospital Research Center, Canada.

\section{Notes and references}

1 A. Atala, D. J. Irvine, M. Moses and S. Shaunak, MRS Bull., 2010, 35.

2 E. Jabbari, J. Leijten, Q. Xu, and A. Khademhosseini. Materials today, 2016, doi:10.1016/j.mattod.2015.10.005

3 O. Buznyk, N. Pasyechnikova, M. M. Islam, S. Iakymenko, P. Fagerholm and M. Griffith, Clin. Transl. Sci., 2015, 8, 558-562.

4 P. Fagerholm, N. S. Lagali, K. Merrett, W. B. Jackson, R. Munger, Y. Liu, J. W. Polarek, M. Soderqvist and M. Griffith, Sci. Transl. Med., 2010, 2, 46ra61.

5 R. Ravichandran, M. Griffith and J. Phopase, J. Mater. Chem. B, 2014, 2, 8466-8478.

6 T. Luo and K. L. Kiick, Eur. Polym. J., 2013, 49, 2998-3009.

7 S. M. Yu, Y. Li and D. Kim, Soft Matter, 2011, 7, 7927-7938.

8 L. E. O'Leary, J. A. Fallas, E. L. Bakota, M. K. Kang and J. D. Hartgerink, Nat. Chem., 2011, 3, 821-828.

9 P. J. Stahl, N. H. Romano, D. Wirtz and S. M. Yu. Biomacromolecules, 2010, 11, 2336-2344.

10 C. M. Perez, A. Panitch and J. Chmielewski, Macromol. Biosci., 2011, 11, 1426-1431.

11 C. M. Rubert Perez, L. A. Rank and J. Chmielewski, Chem. Commun. (Camb), 2014, 50, 8174-8176.

12 A. Y. Wang, S. Leong, Y. C. Liang, R. C. C. Huang, C. S. Chen and S. M. Yu, Biomacromolecules, 2008, 9, $2929-2936$.

13 P. A. Parmara, L. W. Chowa, J-P. St-Pierrea, C-M. Horejsa, Y. Y. Peng, J. A. Werkmeister, J. A. M. Ramshaw and M. M. Stevens, Biomaterials, 2015, 54, 213-225.

14 X. Mo, Y. J. An, C. S. Yun and S. M. Yu, Angew. Chem., Int. Ed., 2006, 45, 2267-2270.

15 M. Griffith and D. G. Harkin, Curr. Opin. Ophthalmol., 2014, 25, 240-247.

16 M. Miotto, R. M. Gouveia and C. J. Connon, J. Funct. Biomater., 2015, 6, 687-707.

17 M. Matsusaki, R. Amekawa, M. Matsumoto, Y. Tanaka, A. Kubota, K. Nishida and M. Akashi, Adv. Mater., 2011, 23, 2957-2961.

18 G. Uzunalli, Z. Soran, T. S. Erkal, Y. S. Dagdas, E. Dinc, A. M. Hondur, K. Bilgihan, B. Aydin, M. O. Guler and A. B. Tekinay, Acta Biomater., 2014, 10, 1156-1166.

19 J. M. Harris and R. B. Chess, Nat. Rev. Drug Discov., 2003, 2, 214-221. 


\section{Supplementary Materials}

Supplementary Methods

Supplementary References

Supplementary Figure 1 In vivo confocal images of pig corneas grafted with

CLP-PEG implants and unoperated control corneas at

12 months post-operation. 


\section{Supplementary Methods}

\section{CLP-PEG production and characterization}

The 38 amino acid long CLP peptide, Cys-Gly-(Pro-Lys-Gly) 4 (Pro-HypGly) ${ }_{4}$ (Asp-Hyp-Gly) ${ }_{4}$, was synthesized on a Symphony automated peptide synthesizer (Protein Technologies Inc., Tucson, AZ, U.S.A.). 2-(6-Chloro-1Hbenzotriazole-1-yl)-1,1,3,3-tetramethylaminium hexafluorophosphate (HCTU) (ChemPep Inc., Wellington, FL, USA) was used as the activating reagent. The 0.1mmol scale synthesis used Fmoc-Gly-PEG-PS resin (Applied Biosystems, Life Technologies Europe BV, Sweden) and amino acids in each coupling. The resulting peptides were cleaved from the resin by treatment with a mixture of trifluoroacetic acid (TFA), water and triisopropylsilane (TIS) $(95: 2.5: 2.5 \mathrm{v} / \mathrm{v} ; 10 \mathrm{~mL}$ per gram of polymer). They were then filtered and the TFA was evaporated. CLP peptide then was precipitated by the addition of cold diethyl ether, centrifuged and lyophilized. Prior to use, CLP was resuspended in milli-Q water.

Aqueous solutions of 8-arm PEG-maleimide and purified CLP were mixed at a molar ratio of PEG-maleimide:CLP (1:5) at room temperature under Good Laboratory Practice (GLP) conditions. After four days of continuous stirring, a homogenous solution of the bio-conjugate was dialyzed using a 12-14 kD MW cut off tubing (Spectrum Laboratories, Inc., CA, US) for 2-3 days to remove the unreacted reactants. After dialysis, the solution was lyophilized and stored at $4{ }^{\circ} \mathrm{C}$ until further use.

The bio-conjugation of CLP with 8-arm PEG Maleimide was characterized using ${ }^{1} \mathrm{H}$ NMR, on an Oxford $300 \mathrm{MHz}$ spectrometer (Varian, CA, US) at room temperature. Briefly 1\% solutions of PEG-maleimide and CLP-PEG were made in 
$\mathrm{C}_{2} \mathrm{D}_{6} \mathrm{OS}$ (Dimethyl- $\mathrm{d}_{6}$ sulfoxide, Armar Chemicals, Döttingen, Switzerland). The resonance of deuterated solvent $\left(\mathrm{C}_{2} \mathrm{D}_{6} \mathrm{OS}, \delta=2.5\right)$ was used as an internal standard.

The triple helical structure of CLP and CLP-PEG were evaluated using Chirascan $^{\mathrm{TM}}$ CD Spectrometer (Applied Photophysics Ltd., Surrey, UK). Briefly, 1\% of sample solutions were prepared and a quartz cell of $0.1 \mathrm{~cm}$ path length was used to record the CD spectra at $180-260 \mathrm{~nm}$ wavelengths, at a scan rate of $1 \mathrm{~nm} / \mathrm{s}$ at room temperature.

\section{CLP-PEG and recombinant human collagen-phosphorylcholine hydrogels}

Unless otherwise stated, all chemicals were purchased from Sigma-Aldrich (MO, USA). A T-piece syringe mixing system we previously described ${ }^{1}$ was used to make CLP-PEG hydrogels. 500mg of $12 \%$ (w/w) CLP-PEG was taken into a $2 \mathrm{ml}$ glass syringe and calculated volumes of N-hydroxysuccinimide (NHS) and N-(3dimethylaminopropyl)-N'-ethylcarbodiimide (EDC) were added to the syringe mixing system. The molar equivalents of CLP-PEG-NH 2 : EDC was 1:2 and the molar ratio of EDC: NHS was 1:1. All reagents were thoroughly mixed prior to moulding the hydrogel into flat sheets or cornea shape implants.

Benchmark hydrogels comprising Recombinant Human Collagen type III (RHC; Fibrogen Inc., San Francisco, CA, USA) with 2-methacryloyloxyethyl phosphorylcholine (MPC; Paramount Fine Chemicals Co. Ltd, Dalian, China) (RHCMPC) were fabricated using our previously published protocol².

\section{Characterisation of hydrogels}

All samples were tested in triplicate. After demoulding, the hydrogels were kept in PBS for 24 hours. The wet weight of hydrogels $\left(\mathrm{W}_{0}\right)$ was obtained after gentle 
blotting on filer paper to remove surface water. The samples were then dried under vacuum until a constant weight (W) was obtained. The total equilibrated water content of the hydrogels $\left(\mathrm{W}_{\mathrm{t}}\right)$ was calculated according to the following equation: $\mathrm{W}_{\mathrm{t}}=\{(\mathrm{W}-$ $\left.\left.\left.\mathrm{W}_{0}\right) / \mathrm{W}\right)\right\} \times 100 \%$

Optical properties such as transmission of white light and back-scattering were measured at room temperature using a custom-built instrument. ${ }^{2}$ The percent transmission of the samples was compared to the open beam intensity. The relative percent of light back scattered from the collimated beam by the sample was measured with a circular array of 8 photodiodes each at 30 degrees off axis.

The mechanical properties of CLP-PEG hydrogels were measured by using an Instron universal test machine (Biopuls 3343, High Wycombe, UK) equipped with Bluehell2 software. The measurements were carried out under water-immersion at $37^{\circ} \mathrm{C}$. Dumb-bell shaped hydrogels of $500 \mu \mathrm{m}$ thick with a gauge length of 14 x $6 \mathrm{~mm}$ and grips at each end of $10 \times 6 \mathrm{~mm}$, were cast in moulds. The mechanical testing was carried out using a 50N load cell with a crosshead speed of $10 \mathrm{~mm} / \mathrm{min}$.

The resistance of CLP-PEG and RHC-MPC hydrogels to collagenase was determined as we previously described. ${ }^{3}$ Clostridium histolyticum (Sigma-Aldrich, St.Louis, USA). Hydrogels were placed in a vial containing $5 \mathrm{U} / \mathrm{mL}$ collagenase from Clostridium histolyticum (Sigma-Aldrich, St.Louis, USA in 0.1M Tris-HCl (pH 7.4) and $5 \mathrm{mM} \mathrm{CaCl} 2$ at $37^{\circ} \mathrm{C}$. The collagenase solution was changed at every 8 hours and the percent residual mass of the sample was measured at different time points.

\section{Subcutaneous implantation in rats}

After approval from the local ethical committee (Linköpings Djurförsöksetiska Nämnd) and in compliance with the Swedish Animal Welfare 
Ordinance and the Animal Welfare Act, CLP-PEG hydrogel samples, $1 \mathrm{~cm}$ diameter $\mathrm{x}$ 500 um thick, were implanted subcutaneously into rats as per ISO 10993-6 to test for local reaction to CLP-PEG hydrogels. Each sample was inserted into a subcutaneous pocket created by blunt dissection in the paravertebral region of the back of 9 week old Wistar rats. A total of 4 samples were evaluated in a total of 4 rats. Implantation sites were checked for healing, skin appearance, and re-growth of hair post-surgery. After 90 days, the rats were euthanized and the implants were harvested and fixed in 4\% paraformaldehyde for histopathological examination following haematoxylin and eosin staining (H\&E).

\section{CLP-PEG implantation and evaluation in mini-pig corneas}

In preparation for clinical translation, in compliance with the OECD Principle of Good Laboratory Practice (GLP), ENV/MC/CHEM (98) 17, 1997, and with local ethical permission from Stockholms Norra Djurförsöksetiska Nämnd, one CLP-PEG implant was grafted into one cornea each of four Gottingen mini-pigs (Ellegaard, Denmark) by anterior lamellar keratoplasty (ALK), at Adlego Biomedical AB (Solna, Sweden). Animals were intubated and anaesthetized prior to surgery. The right cornea of each pig eye was cut with a $6.5 \mathrm{~mm}$ circular trephine to a depth of $500 \mu \mathrm{m}$, and the corneal button was then manually dissected with a diamond knife and removed. Hydrogel implants were cut with a $6.75 \mathrm{~mm}$ diameter trephine and placed into the surgical bed. A piece of clinical human amniotic membrane (HAM) (St:Erik's Eye Hospital, Stockholm) was placed over the implant to suppress undesired inflammation and the implants were kept in place with overlying sutures (10/0 monofilament nylon). Upon completion of the surgery, an antibacterial and antiinflammatory ophthalmic suspension (Tobrasone with $3 \mathrm{mg} / \mathrm{ml}$ dexamethasone and 
1mg/ml tobramycin, Alcon, Sweden) was administered. The maintenance dose was 1 drop, 3 times daily for 5 weeks. The unoperated contralateral corneas and RHC-MPC hydrogels served as controls.

The health status of all animals were monitored throughout the 12 month study. The corneas and implants were evaluated before surgery, at 5 weeks and then at 3, 6, 9 and 12 months after surgery. The examinations were performed by a surgeon who was blinded to which animals received CLP-PEG or RHC-MPC hydrogels. These examinations included slit lamp biomicroscopy (to evaluate haze from a 0 to +4 scale, any neovascularization and general health of the eye), Schrimer's tear test to access tear function, pachymetry to determine any changes in corneal thickness and in vivo confocal microscopy (Heidelberg HRT3 with a Rostock Cornea Module, Heidelberg Engineering GmbH, Dossenheim, Germany) to access in-growth of corneal cells, nerves and any blood vessels or inflammatory cells.

\section{Evaluation of regeneration}

At 12 months post-operation, the mini-pigs were euthanized after their final clinical eye examination. The entire implanted cornea and unoperated contralateral controls were dissected out with 2-3 mm of the surrounding sclera. A central $3 \mathrm{~mm}$ diameter, full-thickness sample was trephined out and snap frozen for collagen analyses. The remaining cornea was divided into pieces, with half fixed in $4 \%$ buffered paraformaldehyde and processed for histopathological examination by a $3^{\text {rd }}$ party certified veterinary pathologist (BioVet AB, Sollentuna, Sweden).

For protein analyses, each frozen $3 \mathrm{~mm}$ diameter sample from operated and control corneas was weighed and re-suspended in $10 \mathrm{mM} \mathrm{HCl}$ containing $1 \mathrm{mg} / \mathrm{mL}$ pepsin. The samples were digested with porcine pepsin at $2-8^{\circ} \mathrm{C}$ for $96 \mathrm{~h}$ and the 
soluble fraction (supernatant) was recovered by centrifugation. An aliquot of the supernatant was mixed with NuPAGE 4X LDS sample buffer (Life Technologies) denatured at $75^{\circ} \mathrm{C}$ for 8 minutes and analyzed on 3-8\% Tris-acetate gels under nonreducing conditions. Proteins were visualized by staining with Gelcode Blue. Stained gels were scanned and band intensity was quantitated using a GE Healthcare ImageQuant350 equipped with ImageQuant TL image analysis software version 7.0.

To determine if the initial CLP-PEG implant had been completely remodeled or was still present within the regenerated neo-corneas, FTIR spectra of CLP and PEG-maleimide alone, and non-implanted CLP-PEG hydrogel was compared to cornea samples from CLP-PEG implanted eyes and control eyes. The samples were scanned between 4000 to $400 \mathrm{~cm}^{-1}$ using VERTEX 70 FTIR spectrometer (Bruker, Billerica, MA, USA) at the resolution of $4 \mathrm{~cm}^{-1}$ averaging 200 scans.

\section{Supplementary References}

1. M. M. Islam, M. Griffith and K. Merrett, Methods Mol. Biol., 2013, 1014, 157-164.

2. D. Priest and R. Munger, Ophthalmol. Vis. Sci., 1998, 39, S352.

3. C. Deng, F. Li, J. M. Hackett, S. H. Chaudhry, F. N. Toll, B. Toye, W. Hodge and M. Griffith, Acta Biomater., 2010, 6, 187-194. 


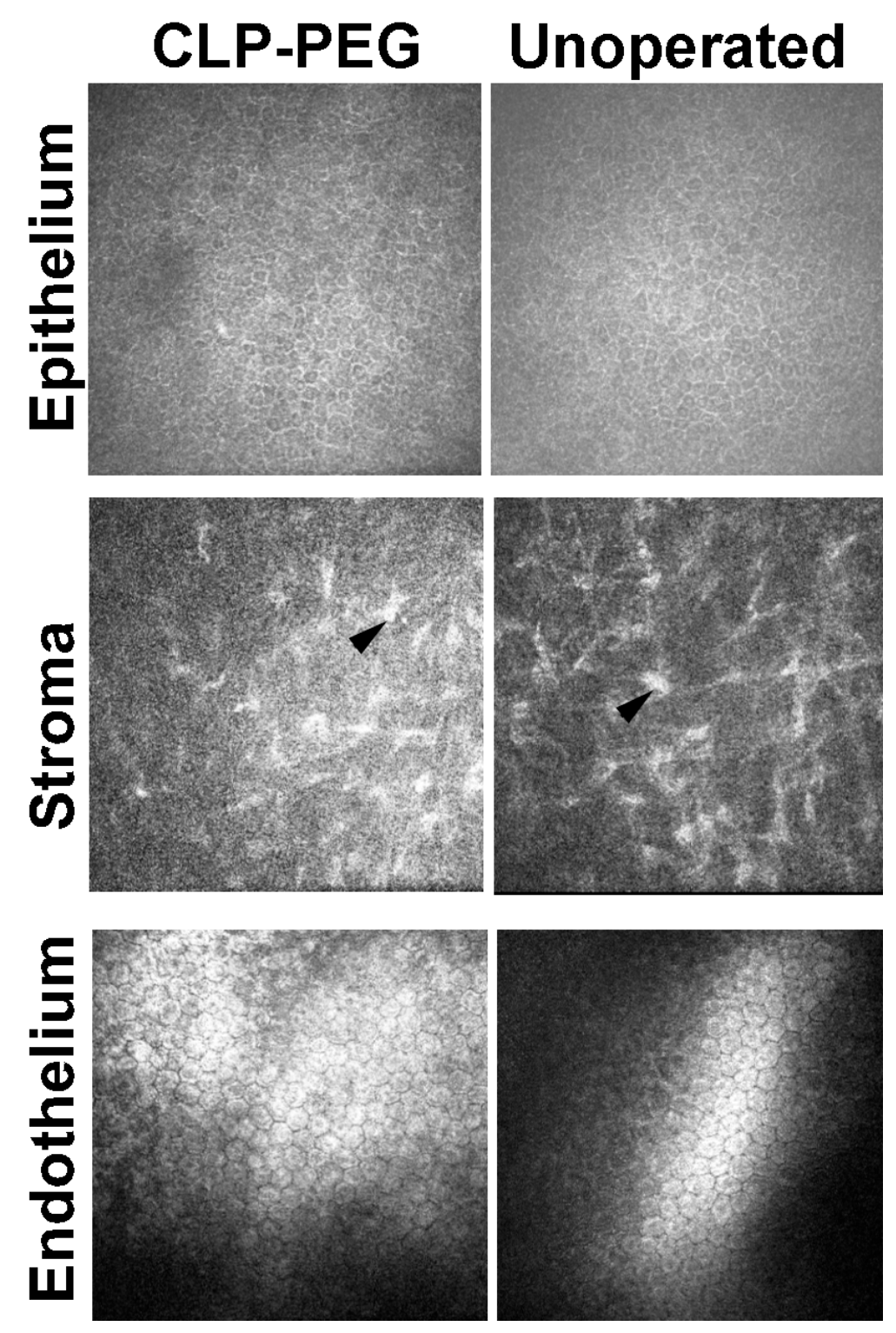

Supplementary Fig. 1: In vivo confocal microscopic images of pig corneas grafted with CLP-PEG implants and unoperated control corneas at 12 months post-operation. Epithelial coverage and stromal cell ingrowth have resulted in neo-corneal tissues similar to the natural, unoperated cornea. Arrowheads indicate stromal cells. The endothelium was left intact during the surgery. 